\title{
CAUSAS DE DESMAME PRECOCE NO BRASIL
}

\author{
Cibele de Moura Sales ${ }^{1}$, Sandra Cristina Seixas ${ }^{2}$
}

RESUMO: Pesquisa bibliográfica com o objetivo de identificar causas de desmame precoce no Brasil. Foram selecionados 16 artigos de pesquisa, encontrados a partir de buscas na Biblioteca Virtual de Saúde (BVS), em bases de dados da Bireme, SciELO, Medline e Lilacs. Os fatores relacionados com o desmame precoce encontrados foram: uso da chupeta, hospitalização da criança, escolaridade materna e paterna, sintomas depressivos da mãe, influência das avós, intercorrências nas mamas no puerpério, crenças e valores das mães, entre outros. Faz-se necessária a detecção precoce dos fatores de risco à interrupção do aleitamento materno, para aconselhamento e manejo clínico adequados, bem como o acompanhamento pelos profissionais de saúde do binômio mãe/filho durante o período da amamentação exclusiva.

PALAVRAS-CHAVE: Aleitamento materno; Desmame precoce; Amamentação exclusiva.

\section{CAUSES OF EARLY WEANING IN BRAZIL}

\begin{abstract}
Literature review aiming to identify the causes of early weaning in Brazil. Sixteen research articles were selected after search in the Virtual Health Library, Bireme, SciELO, Medline and Lilacs databases. The factors related to early weaning were: use of pacifier, child's hospitalization, parents' educational level, mothers' depressive symptoms, grandmothers' influence, breast complications in puerperium, mothers' beliefs and values, among others. Early detection of risk factors to breastfeeding interruption is necessary for guidance and appropriate clinical management as well as the monitoring by health professionals of mother/son dyad during the period of exclusive breastfeeding.
\end{abstract}

KEYWORDS: Breastfeeding, Early weaning, Exclusive breastfeeding.

\section{CAUSAS DE DESTETE PRECOZ EN BRASIL}

RESUMEN: Se trata de una investigación bibliográfica con el objetivo de identificar y agrupar las causas del destete precoz en el país. Fueron seleccionados 16 artículos de investigación encontrados a partir de investigación en la biblioteca virtual de salud, la BVS, en bases de datos de la Bireme, SciELO, Medline y Lilacs. Los factores relacionados con el destete precoz encontrados fueron uso del chupete, hospitalización del niño, escolaridad materna y paterna, síntomas depresivos de la madre, influencia de las abuelas, alteraciones de las mamas en el puerperio, creencias y valores de las madres, entre otros. Se hace necesaria a detección precoz de los factores de riesgo a la interrupción de la lactancia materna precozmente para aconsejamiento y manejo clínico adecuados, así como el acompañamiento por los profesionales de salud del binomio madre/hijo durante el periodo de la lactancia exclusiva.

PALABRAS CLAVE: Lactancia materna, Destete precoz, Lactancia exclusiva.

${ }^{1}$ Enfermeira. Docente da Universidade Estadual de Mato Grosso do Sul. Especialista em Enfermagem Médico-Cirúrgica pela Faculdade de Enfermagem do Hospital Israelita Albert Einstein na modalidade de residência de enfermagem. Mestre em Saúde Coletiva pela Universidade Federal de Mato Grosso do Sul. Doutoranda em Ciências da Saúde pela Universidade de Brasília-UnB.

${ }^{2}$ Enfermeira da rede municipal de Campo Grande-Mato Grosso do Sul. Especialista em Saúde da Família pela Escola Nacional de Saúde Pública-ENSP na modalidade de residência.

Autor correspondente:

Cibele de Moura Sales

Rua Acaia, 189 - 79097-230 - Campo Grande-MS

Recebido: 30/07/07

E-mail: cm.sales@terra.com.br

Aprovado: 21/12/07

Cogitare Enferm 2008 Jul/Set; 13(3):443-7 


\section{INTRODUÇÃO}

Define-se aleitamento materno ou amamentação quando as crianças recebem leite humano, com ou sem alimentos complementares de qualquer natureza. Estão em amamentação exclusiva as crianças que recebem somente leite humano, diretamente da mama ou extraído, e nenhum outro líquido ou sólido, com exceção de medicamentos ${ }^{(1)}$. A recomendação da Assembléia Mundial de Saúde de 1994 é de que a amamentação exclusiva é o alimento ótimo para lactantes de zero a seis meses, apontando para a não necessidade e a inconveniência de introduzir precocemente outros alimentos na dieta infantil. A introdução precoce de líquidos ou sólidos na dieta infantil, antes dos seis meses de idade, é designada desmame precoce $^{(2)}$.

O editorial da revista Lancet, do mês de novembro de 1994, afirma:

\begin{abstract}
Se uma nova vacina, capaz de prevenir um milhão ou mais de mortes infantis por ano, barata, segura, de uso oral, sem necessidade de cadeia de frio estivesse disponível, ela se tornaria uma exigência imediata da saúde pública. A amamentação oferece todos estes requisitos e muito mais, porém ela necessita de uma "cadeia de calor" - isto é, assistência adequada para construir a autoconfiança materna e proteção contra as práticas prejudiciais $^{(3: 1239-40)}$.
\end{abstract}

A amamentação exclusiva confere proteção contra infecções como diarréias, infecções neonatais em maternidade, infecções respiratórias, otites, infecções bacterianas entre outras. $\mathrm{O}$ efeito protetor do leite contra diarréias, por exemplo, pode diminuir substancialmente, quando a criança recebe além do leite materno, outro alimento. A importância dessa informação decorre do fato de no Brasil, a diarréia ser uma das principais causas de mortalidade infantil ${ }^{(4)}$.

Além disso, a ingestão precoce de alimentos na dieta da criança pode ser prejudicial, já que o seu sistema digestivo e rins são imaturos, tendo dificuldades no manejo de metabólicos diferentes do leite humano, podendo causar reações de hipersensibilidade e eliminação de solutos de forma adequada ${ }^{(4)}$.

Outras vantagens do aleitamento materno são apontadas por diversos autores ao afirmar que se trata de um alimento natural, economicamente barato, não contaminado por bactérias, é fresco e prontamente disponível na temperatura adequada e se constitui um método prático de alimentar a criança. O benefício psicológico também é reforçado durante a amamentação, pois é através dela que se pode estabelecer uma profunda relação entre o binômio mãe/ filho, determinada por um processo de interação e proporcionada por fortes estímulos sensoriais, auditivos, táteis, visuais e emocionais ${ }^{(5-11)}$. O aleitamento materno proporciona crescimento e desenvolvimento saudável promovendo o desenvolvimento sensor e cognitivo da criança ${ }^{(5,8-9,12)}$. Apresenta também vantagens para a saúde materna, dentre elas: a sua atuação como um método contraceptivo natural, o restabelecimento pósparto mais rápido e como um fator de proteção na prevenção do câncer de mama ${ }^{(6,12)}$.

Apesar das diversas vantagens da amamentação exclusiva, apenas aproximadamente $38 \%$ dos recém-nascidos brasileiros recebem exclusivamente leite materno entre zero e quatro meses de idade ${ }^{(5,13)}$. A amamentação é influenciada pela sociedade e condições da vida da mulher e se dá dentro de um contexto sociocultural. Dessa forma, também é um comportamento social passível de mudanças de acordo com as épocas e os costumes ${ }^{(14-15)}$. Sendo o aleitamento materno um comportamento social mutável, faz-se necessário conhecer as causas atuais de desmame precoce para proposição de intervenções adequadas pelo serviço e profissionais de saúde. Assim, neste artigo o objetivo foi identificar causas de desmame precoce no Brasil.

\section{DESCRIÇÃO DO MÉTODO}

Trata-se de uma pesquisa bibliográfica para a qual se utilizou textos publicados em periódicos de 1997 a 2008, que tratavam sobre o aleitamento materno até o sexto mês de vida da criança. A busca foi realizada em artigos de revistas científicas extraídas via Internet, nos bancos da Bireme, SciELO, Lilacs e Medline .

As seguintes combinações de unitermos foram utilizadas: amamentar, amamentação, leite materno, leite humano, aleitamento materno, desmame. Encontrou-se 32 artigos que tratavam de questões relacionadas com o aleitamento materno. Realizou-se uma revisão dos títulos e resumos a fim de selecionar os artigos a serem lidos integralmente. Foram incluídos os artigos que apresentavam dados de pesquisa sobre as causas de desmame precoce, num total de 15 artigos selecionados. Os dados foram organizados de acordo com as causas de desmame precoce encontradas na literatura, os identificados como fatores independentes, foram descritos sozinhos e os fatores interdependentes são apresentados de forma associada. 


\section{RESULTADOS E DISCUSSÃO}

No estudo ${ }^{(16)}$ foi verificada uma significância estatística entre uso de chupeta e uma menor duração do aleitamento materno. É destacado que apesar de não ser possível comprovar o efeito de causalidade (confusão no modo de sucção e/ou diminuição das mamadas), pode-se relacionar com a presença de problemas no aleitamento materno como dificuldades e motivação reduzida para amamentar. Dessa forma a introdução de chupetas para crianças nas primeiras semanas de vida deve servir de alerta aos profissionais de saúde, de que as mães estão necessitando, de apoio e orientações adequados ${ }^{(16-18)}$.

A maior escolaridade paterna está relacionada com menor duração do aleitamento materno. Existem vários estudos que observam a duração maior do hábito nas crianças de nível socioeconômico mais baixo, principalmente em países em desenvolvimento. A escolaridade paterna aqui representa uma condição social e econômica melhor ${ }^{(16)}$.

Existe relação entre o pai residir com a criança e uma maior duração do aleitamento materno ${ }^{(16)}$. Um estudo $^{(19)}$ demonstra que quando os pais aprovavam intensamente a amamentação $98,1 \%$ das crianças eram amamentadas, enquanto que apenas $26,9 \%$ o eram quando os pais eram indiferentes. Os pesquisadores alertam para o fato de que a influência paterna é subestimada pelos profissionais e serviços de saúde, tanto que não se preocupam em preparar os futuros pais e envolvê-los nos grupos operativos, consultas de pré-natal entre outras. Esses resultados apontam para a necessidade de um maior envolvimento dos pais no acompanhamento da saúde da criança desde a gestação(16,19-20).

A hospitalização pode levar ao desmame de até $60 \%$ dos lactentes que estavam amamentando no início da internação. Interessante que o desmame não foi associado ao tempo de duração da internação e nem por questões médicas. Mesmo crianças que tiveram internadas por curtos períodos de tempo, tiveram o aleitamento materno exclusivo suspenso. Vários fatores podem estar relacionados, a falta de uma rotina específica, de práticas de incentivo ao aleitamento por parte do hospital, de sensibilização dos profissionais de saúde envolvidos, dificuldades de ordem física, desconforto das acomodações para as mães, dificuldade das mães de permanecerem tempo integral no hospital, domicílio distante do hospital e falta de banco de leite nos hospitais. Os autores sugerem que o trabalho de incentivo ao aleitamento materno realizado nas maternidades deve ser estendido a todas as unidades que prestam assistência a lactantes ${ }^{(21)}$.

Apesar de a literatura apresentar uma associação negativa entre aleitamento materno e escolaridade materna em países em desenvolvimento, alguns estudos no Brasil apontam para uma relação positiva. Porquanto, as mães seguiam o modelo dos países desenvolvidos, ou seja, aquelas de maior escolaridade amamentaram por maior tempo seus filhos. Outro dado interessante e que provavelmente está associado à escolaridade é o fato de que as mães que trabalhavam fora de casa amamentaram por mais tempo. Ao contrário do esperado pelos autores, o tempo de acompanhamento da criança pelo posto de saúde não influenciou o tempo de amamentação. Os autores levantam a hipótese de que as mulheres mais instruídas estão em fase de valorização do aleitamento materno exclusivo, enquanto as demais ainda não foram sensibilizadas ${ }^{(22-23)}$.

No estudo ${ }^{(24)}$ encontrou-se relação entre sintomas depressivos da mãe e duração e freqüência de amamentação exclusiva. O percentual de mães com sintomas depressivos, $35,7 \%$, demonstra a gravidade do problema. Os autores também apresentam um estudo realizado na Austrália que associa depressão ao desmame precoce, afirmando que quanto mais cedo ocorre o episódio depressivo, menor o tempo de amamentação, destacando o impacto negativo da depressão sobre o engajamento materno no aleitamento.

A atenção à saúde mental das mulheres deve ser maior, considerando a alta prevalência de sintomas depressivos no pós-parto e sua relação com o desmame precoce e cuidados com o bebê( ${ }^{(24)}$.

O fato de a mãe considerar seu leite como fraco, insuficiente ou que a qualidade do mesmo deixa a desejar, associados com o choro e a fome do bebê são verdadeiros condicionantes para a interrupção da amamentação exclusiva ${ }^{(6,10,25-27)}$. A mãe também pode considerar o seu bebê guloso e que é preciso um "super leite" para dar conta das necessidades alimentares do mesmo, sentindo-se incapaz de produzi-lo ${ }^{(28)}$.

As intercorrências da mama no puerpério, como fissuras, ingurgitamento mamário e a dor também influenciam negativamente na duração da amamentação exclusiva. As mães, que realizaram cesarianas, apresentam maior dificuldade para amamentar, devido ao próprio processo cirúrgico que sofreram ${ }^{(12,29)}$.

Existem alguns fatores relacionados à própria característica da mulher-mãe-puérpera. Diversos autores relatam que as experiências vivenciadas pela 
mulher terão influência na continuidade da amamentação. Deve-se considerar a experiência particular de cada puérpera, questionar quais situações ela pôde presenciar ao longo da vida. É importante lembrar que a amamentação é culturalmente determinada, isto é, passada de mãe para filha: as mães que amamentaram seus filhos, também terão seus netos amamentados $^{(9-10,25,27)}$. Foi identificado que as avós influenciam tanto positiva como negativamente na amamentação, na duração e na sua exclusividade. Segundo essa pesquisa pouco mais da metade das avós aconselharam o uso de água ou chá, e ainda algumas (17\%) tomaram a iniciativa de oferecer líquidos às crianças ${ }^{(30)}$.

Propõe-se ${ }^{(27)}$ ações que privilegiem a introjeção de novos valores culturais em favor da amamentação, valores esses que considerem a amamentação como um ato a ser aprendido e protegido pela sociedade.

As condições de vida precárias também estão associadas com o a interrupção do aleitamento materno exclusivo. Crianças de condições de vida muito baixa têm 2,5 vezes mais chances de serem desmamadas precocemente, bem como filhos de mulheres menores de 20 anos que apresentam um risco 2,2 vezes maior de serem desmamadas quando comparado aos filhos de mães que apresentam idade entre 20 e 34 anos $^{(31)}$.

Sabendo dos benefícios que o aleitamento materno exclusivo traz ao binômio, mãe/filho e conhecendo os fatores que podem levar ao desmame precoce, faz-se necessário, a detecção precoce dos riscos presentes para a elaboração de ações preventivas ao desmame precoce. Um estudo ${ }^{(32)}$ propõe a utilização de um protocolo logo após o nascimento para a identificação dos binômios com maiores dificuldades, através de escores, e assim um membro da equipe, treinado em manejo clínico e aconselhamento da amamentação, passaria a atuar, e sendo útil para os critérios de alta hospitalar. Outras ações podem ser pensadas e estudadas com o escopo de diminuir o alto índice de desmame precoce.

$\mathrm{O}$ respeito ao saber do outro, o bom senso, o comprometimento e competência profissional, a reflexão crítica sobre a prática, o saber escutar e a convicção de que a mudança é possível é a chave para o recomeço. Sendo ele o resgate da percepção sobre amamentar, o reconhecimento da relação da comunicação no processo de trabalho em saúde e sua possibilidade como ferramenta de transformação social ${ }^{(26)}$.

\section{CONCLUSÃO}

Muitos são os fatores relacionados com o desmame precoce, alguns com vários estudos e outros, como a relação com os sintomas depressivos e a influência paterna, com poucos estudos no Brasil. Existe associação entre menor período de amamentação exclusiva e uso de chupetas, escolaridade paterna e materna, condições de vida precárias, as crenças da mãe sobre o leite materno, intercorrências das mamas no puerpério, influência das avós e influência cultural. A importância de conhecer, esses fatores decorre do benefício da amamentação ao binômio, mãe/filho e dos prejuízos do desmame precoce. Com o conhecimento é possível a detecção precoce do serviço e dos profissionais de saúde e assim planejar intervenções que promovam e facilitem o aleitamento materno exclusivo.

\section{REFERÊNCIAS}

1 OPAS. Ministério da Saúde. Reunião sobre aleitamento materno: 14-15 de abril de 1999. Brasília, 1999.

2 WHO. Infant and young child nutrition. IN: World Assembly Resolution, $47^{\circ}$ Geneva. 1994.

3 A warm chain for breastfeeding. Editorial. Lancet, 1994; 344(8932):1239-40.

4 Giugliani ERJ, Victora CG. Normas alimentares para crianças brasileiras menores de dois anos. Embasamento científico. Brasília: OMS; 1997.

5 OPAS/OMS. Amamentação. [acesso em 2004 Mar 26]. Disponível em: www.opas.org.br

6 Sandre-Pereira S, Colares LGT, Do Carmo MGT; Soares EA. Conhecimentos maternos sobre amamentação entre puérperas inscritas no programa de pré-natal. Cad Saúde Públ. 2000;16(2):457-66.

7 Benavides LP, Ponce TE. Practica de la lactancia materna y su relación con la educacion y motivación sobre la lactancia natural en madres que asisten al control infantil en el centro de salud de Ibarra, Ibarra, 1988. Ibarra: Escuela Nacional de Enfermaria; 1989.

8 De Ornes CM, Fernandes MPJ. Capacitación a distancia en lactancia materna. Rev Fac Med. (Caracas) 2000;25(1):100-03.

9 Ichisato SMT, Shimo AKK. Revisitando o desmame precoce através de recortes da história. Rev Latino-Am Enferm. 2002;10(4):578-85. 
10 Nakamura SS, Veiga FF, Ferrarese SRB, Martinez FE. Percepção e conhecimento de meninas escolares sobre o aleitamento materno. J Ped. 2003;79(2):181-8.

11 Nascimento MBR, Issler H. Breastfeeding: making the difference in the development, health and nutrition of term and preterm newsborns. Rev Hosp Clin. 2003;58(1):49-60.

12 Nakano AMS. As vivências da amamentação para um grupo de mulheres: nos limites de ser "o corpo para o filho” e de ser “o corpo para si”. Cad Saúde Publ. 2003;19 (Sup 2):355-63.

13 Camilo DF, Carvalho RVB, Oliveira EF, Moura EC. Prevalência da amamentação em crianças menores de dois anos vacinadas no centro de saúde escola. Rev Nutr Campinas. 2004;17(1):29-36.

14 Silva AAM. Amamentação: fardo ou desejo? Estudo histórico social dos deveres e práticas sobre aleitamento materno na sociedade brasileira [dissertação]. Ribeirão Preto (SP): Escola de Enfermagem de Ribeirão Preto. Universidade de São Paulo; 1990.

15 Bitar MAF. Aleitamento materno: um estudo etnográfico sobre costumes, crenças e tabus ligados a esta prática [dissertação]. Belém (PA): Centro de Ciências da Saúde. Departamento de Enfermagem. Universidade Federal do Pará; 1995.

16 Silveira, FJF, Lamounier JA. Fatores associados à duração do aleitamento materno em três municípios da região do Alto Jequitinhonha, Minas Gerais, Brasil. Cad Saúde Públ. 2006; 22(1):69-77.

17 Audi CAF, Corrêa AMS, Latorre MRDO. Alimentos complementares e fatores associados ao aleitamento materno e ao aleitamento materno exclusivo em lactentes até 12 meses de vida, em Itapira, São Paulo, 1999. Rev Bras Saúde Matern Infant. 2003;3(1):85-93.

18 Soares MEM, Guigiani ERJ, Braur ML, Salgado ACN, Oliveira AP, Aguiar PR. Uso de chupeta e sua relação com o desmame precoce em população de crianças nascidas em hospital amigo da criança. J Ped. 2003;79(4):309-16.

19 Littman H, Medendorp SV, Goldfarb J. The decision to breastfeed: the importance of fathers' approval. Clin Pediatr. 1994;33:214-9.

20 Carrascoza KC, Costa Júnior AL, Moraes ABA. Fatores que influenciam o desmame precoce e a extensão do aleitamento materno. Estudos Psicol. 2005;22(4):4333-440.
21 Souza EL, Silva LR, Sá ACS, Bastos CM, Diniz AB, Mendes CMC. Impacto da internação na prática do aleitamento materno em hospital pediátrico de Salvador, Bahia, Brasil. Cad Saúde Públ. 2008;24(5):1062-70.

22 Escobar AMU et al. Aleitamento materno e condições sócio-econômico-culturais: fatores que levam ao desmame precoce. Rev Bras Saúde Matern Infant. 2002;2(3):253-61.

23 Volpini CCA, Moura EC. Determinantes do desmame precoce no distrito noroeste de Campinas. Rev Nutr. 2005;18(3):311-9.

24 Vitolo MR, Benetti SPC, Bortolini GA, Graeff A, Drachler ML. Depressão e suas implicações no aleitamento materno. Rev Psiq RS. 2007;29(1):28-34.

25 Rezende MA, Sigaud CHS, Veríssimo MR, Chiesa AM, Bertolozzi MR. O processo de comunicação na promoção do aleitamento materno. Rev Latino-Am Enferm. 2002;10(2):234-8.

26 Machado LV, Larocca LM. Intercorrências mamárias e desmame precoce - uma abordagem comunicacional. Cogitare Enferm. 2004; 9(2):89-98.

27 Ramos CV, Almeida JAG. Alegações maternas para o desmame: estudo qualitativo. J Ped. 2003; 79(5):385-90.

28 Borges ALV, Philippi ST. Opinião das mulheres de uma unidade de saúde da família sobre a quantidade de leite materno produzido. Rev Latino-Am Enferm. 2003;11(3):287-92.

29 Rocha SMM, Simpionato E, Mello DB. Apego mãe-filho: estudo comparativo entre mães de parto normal e cesárea. Rev Bras Enferm. 2003:56(2):125-9.

30 Susin LRO, Giugliani ERJ, Kummer SC. Influência das avós na prática do aleitamento materno. Rev Saúde Públ. 2005;39(2):141-7.

31 Oliveira LPM, Assis AMO, Gomes GSS, Prado MS, Barreto ML. Duração do aleitamento materno, regime alimentar e fatores associados, segundo condições de vida em Salvador, Bahia, Brasil. Cad Saúde Pub. 2005;21(5):1519-30.

32 Carvalhaes MABL, Correa CRH. Identificação de dificuldades no início do aleitamento materno mediante aplicação de protocolo. J Ped. 2003;79(1):13-20. 